\title{
Effects of Liquid Helium Bubble Formation in a Superconducting Cavity Cryogenic System
}

\author{
X. Chang, E. Wang, T. Xin
}

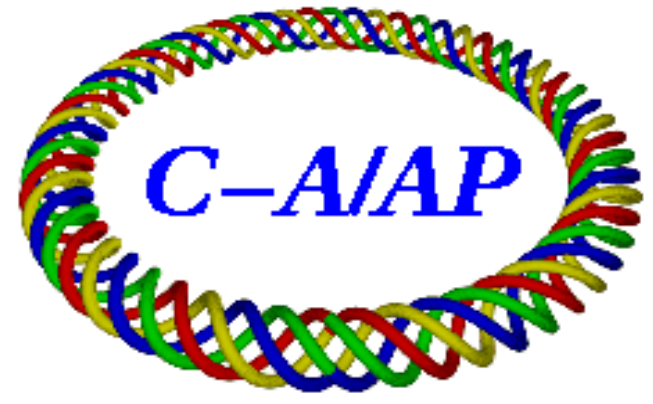

\section{Collider-Accelerator Department Brookhaven National Laboratory Upton, NY 11973}

Notice: This document has been authorized by employees of Brookhaven Science Associates, LLC under Contract No. DE-AC02-98CH10886 with the U.S. Department of En ergy. The United States Government retains a nonexclusive, paid-up, irrevocable, world-wide license to publish or reproduce the published form of this document, or allow others to do so, for United States Government purposes. 


\title{
Effects of liquid helium bubble formation in a superconducting cavity cryogenic system
}

\author{
Xiangyun Chang, ${ }^{1}$ Erdong Wang, ${ }^{2}$ Tianmu Xin, ${ }^{3}$ \\ ${ }^{1}$ Collider Accelerator Department, Brookhaven National Laboratory, Upton, NY 11973 \\ ${ }^{2}$ Physics School, Peking University, Beijing, China, 100081 \\ ${ }^{3}$ Physics and Astronomy Department, Stony Brook University, Stony Brook NY 11974
}

\begin{abstract}
:
We constructed a simple prototype model based on the geometry of the $56 \mathrm{MHz}$ superconducting cavity for RHIC. We studied the formation, in this prototype, of bubbles of liquid helium and their thermal effects on the cavity. We found that due to the low viscosity of the liquid helium, and its small surface tension, no large bubbles formed. The tiny bubbles, generated from most of the area, behaved like light gas travelling in a free space and escaped from the trapping region. The bubbles that were generated in the trapping area, due to its descending geometry, are much bigger than the other bubbles, but due to the liquid flow generated by heating, they still are negligible compared to the size of the trapping region. We expected that the effects of bubbles in our $56 \mathrm{MHz}$ cavity during operation might well be negligible.

Introduction:
\end{abstract}

The configuration of the $56 \mathrm{MHz}$ superconducting cavity for RHIC (Fig.1) encompasses of a deep, horizontal concave area in the liquid helium (LH) space. LH bubbles could be trapped in it, and form big bubble on top of this concave space during operation.

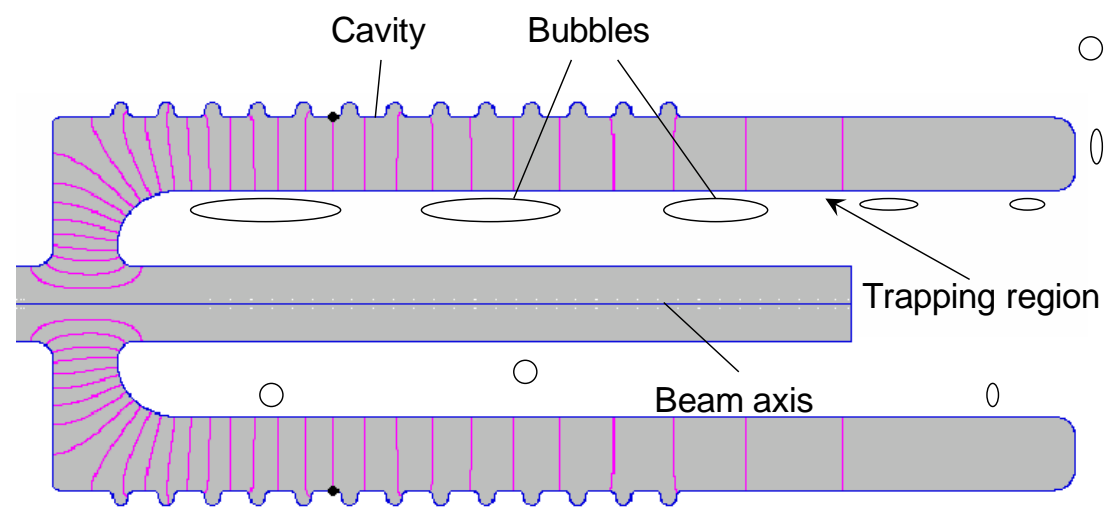

Figure 1: Schematic plot of the RHIC $56 \mathrm{MHz}$ cavity, and the likely region wherein big bubbles accumulate

In our simplified bubble-trapping model, we assumed that once big bubbles formed near the top surface of the cavity, the bubble-covered area is isolated from the LH's heat bath where the temperature is 
assumed to be $4.2 \mathrm{~K}$. The RF heat transfer from this bubble-covered area is mainly through the niobium (Nb) wall. However, this model is not completely accurate because the thermal conductivity of helium gas is about $1 / 3$ that of $\mathrm{LH}$, so that part of the heat may be transferred through the bubble. However, this will not affect our conclusions.

We first estimated the increase in temperature based on our model of the cavity. As shown in fig.2, left plot, the bubble is assumed to be inside the circle with radius $r$. The temperature increase, $d T$, due to radius increase, $\mathrm{dr}$, is

$d T=\frac{\pi r^{2} P_{0} d r}{k A}=\frac{P_{0}}{4 k t} d r^{2}$

where $\mathrm{P}_{0}$ is the RF power density of the $56 \mathrm{MHz}$ cavity, $k \approx 80 \mathrm{~W} / \mathrm{mK}$ is the thermal conductivity of $\mathrm{Nb}$ at LH temperature, $A=2 \pi r t$ is the cross-section of the heat flow, and $t=3 \mathrm{~mm}$ is the thickness of $\mathrm{Nb}$.
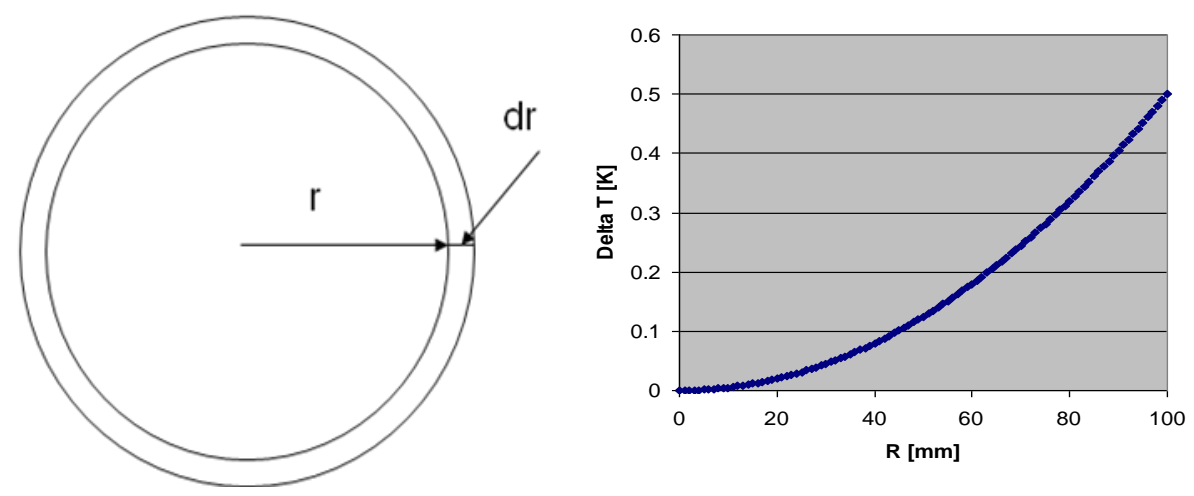

Figure 2: Left plot: RF heat transfer with a round bubble. The circle's edge is the bubble's edge. Right plot: Center-edge temperature difference as a function of the bubble's radius.

From equation (1) we find the temperature difference between the center and the edge of the bubble, $\Delta \mathrm{T}$ :

$\Delta T[K]=\frac{P_{0}}{4 k t} r^{2} \approx 50 r[m]$

The right diagram in fig. 2 plots $\Delta \mathrm{T}$ as a function of the bubble's radius where $P_{0}^{2} \approx 40 \mathrm{~W} / \mathrm{m}^{2}$ is the peak power density at end of the cavity at operation. We see that when the bubble's radius as large as $10 \mathrm{~cm}$, the temperature difference is only about $0.5 \mathrm{~K}$.

Our tests were designed to demonstrate how much the temperature changes due to the formation of bubbles during the operation of the $56 \mathrm{MHz}$ superconducting cavity. Figure 3 illustrates our experimental set-up.

Test setup: 


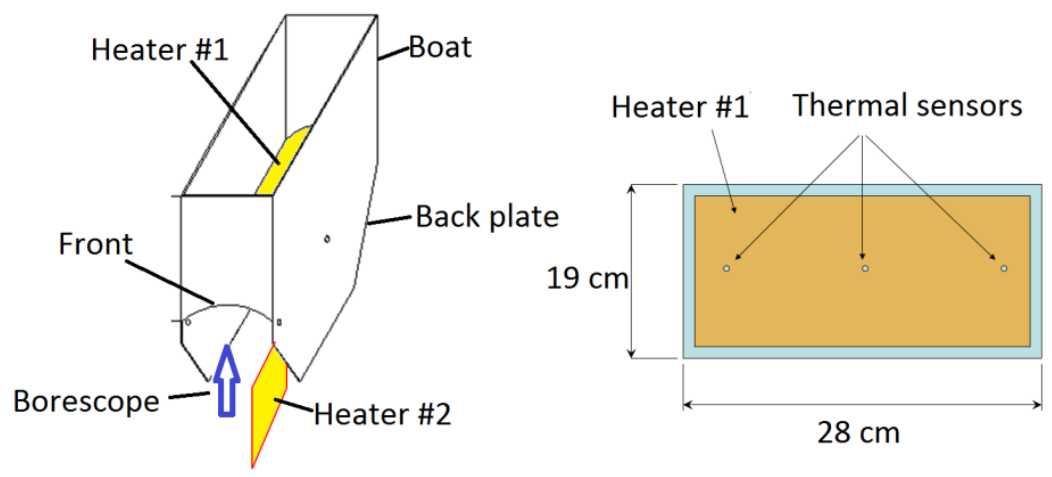

Figure 3: Left: Aluminum boat-shaped prototype. Right: Top view of the boat's bottom plate.

We chose Aluminum 1100 as the prototype material because its thermal conductivity $(\sim 70 \mathrm{~W} / \mathrm{mK})$ is very close to that of $\mathrm{Nb}(\sim 80 \mathrm{~W} / \mathrm{mK})$ at $4.2 \mathrm{~K}$. The boat's bottom plate was swept downwards with the same degree of curvature as that of the $56 \mathrm{MHz}$ trapping cavity.

Heater \#1 and three thermal sensors were cryogenically glued on top side of the boat's bottom. This heater raised the temperature of the bottom plate uniformly with a power density of $40 \mathrm{~W} / \mathrm{m}^{2}$, the peak power density at the end of the $56 \mathrm{MHz}$ cavity. We controlled the level of the LH such that it was lower than boat edges during LH filling and, hence, LH had no direct contact with heater \#1 and the thermal sensors.

Heater \#2, located beneath the bottom plate, was submerged in LH during the tests. Its total power was less than $40 \mathrm{~W}$, the total power of the $56 \mathrm{MHz}$ cavity during operation.

The back plate (not shown) prevented the outward movement of the bubbles. A borescope was placed at the front (bubble exit) below the boat. It pointed upward to gain a worm's-eye view at the bubbles' exit and also was submerged in LH during the tests.

We could adjust manually the angle of the boat's orientation. The $56 \mathrm{MHz}$ cavity will be operated in horizontal orientation. We were able to push down the boat's front to trap more bubbles, and vice versa.

One of the questions we asked ourselves during the tests was whether the readings from the thermal sensors that we placed on top side of the bottom plate (corresponding to the vacuum side in the real cavity) reflect the temperature on the LH side. The answer was an unequivocal yes. As shown in fig.4, the temperature difference on the two sides of the metal plate $\Delta T_{\perp}$ is

$$
\Delta T_{\perp}=P_{\perp} t / k
$$

where $P_{\perp}$ is the power-flow density perpendicular to the surface. Assuming that the vertical heat power density is $P_{\perp}=P_{0}=40 \mathrm{~W} / \mathrm{m}^{2}$, then $\Delta T_{\perp} \approx 10^{-3} \mathrm{~K}$. Therefore, the temperature difference is negligible. In fact, if we had placed the sensors on the LH side, it would have been very hard to obtain the real temperatures because the LH could strongly affect the sensors due to their direct contact with it. 


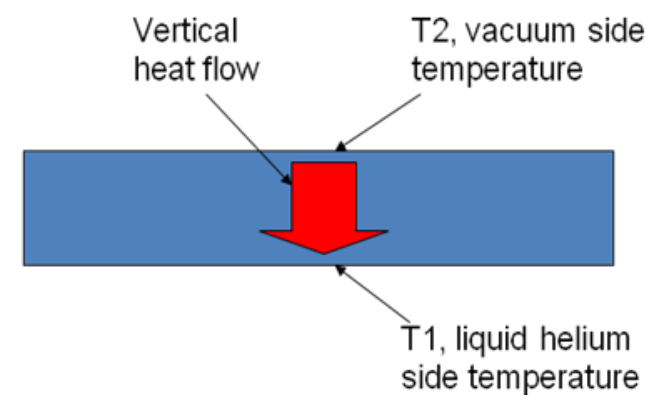

Figure 4: The temperature difference from both side surfaces $\left(\left|T_{2}-T_{1}\right|\right)$ is less than $10^{-3}$

Test results and discussion:

The boat was installed in a Dewar flask that gradually was filled with LH until its level was about $18 \mathrm{~cm}$ above the boat's bottom. We then turned on or off the heaters independently, adjusted that trapping angle, as required for a particular test. As mentioned, when heater \#1 was powered it generated $2 \mathrm{~W}$, such that the power density was $40 \mathrm{~W} / \mathrm{m}^{2}$ on the boat's bottom. When heater \#2 was powered it normally generated $20 \mathrm{~W}$; we adjusted this value from $2 \mathrm{~W}$ to $30 \mathrm{~W}$ at end of the test. The temperature data was acquired at frequency of about $0.5 \mathrm{~Hz}$. The view from the borescope at the boat's exit was video-recorded. Fig. 5 shows the first series of experiment data we obtained.

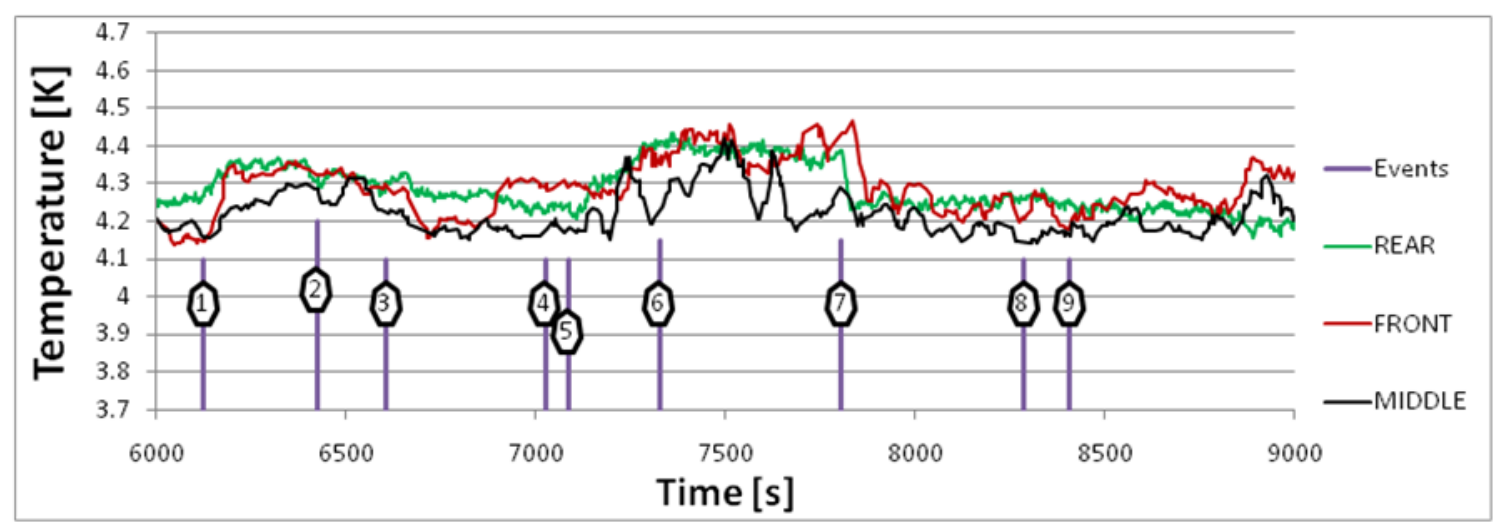

\begin{tabular}{|l|c|c|c|c|c|c|c|c|c|}
\hline Event Number & 1 & 2 & 3 & 4 & 5 & 6 & 7 & 8 & 9 \\
\hline Base heater (\#1) & On & On & & & On & On & & & \\
\hline Bubble heater (\#2) & & On & & & & On & & & \\
\hline Angle & $0^{\circ}$ & $0^{\circ}$ & $0^{\circ}$ & $10^{\circ}$ & $10^{\circ}$ & $10^{\circ}$ & $10^{\circ}$ & $10^{\circ}$ & $-10^{\circ}$ \\
\hline \multicolumn{7}{|c|}{ Trap } \\
\hline
\end{tabular}

Figure 5: $1^{\text {st }}$ series of experiment data. The curves are the temperature variations of the front sensor, middle sensor, and rear sensor during the test. The event number indicates the new actions being taken in the process. The table in the plot lists the conditions during each event. 


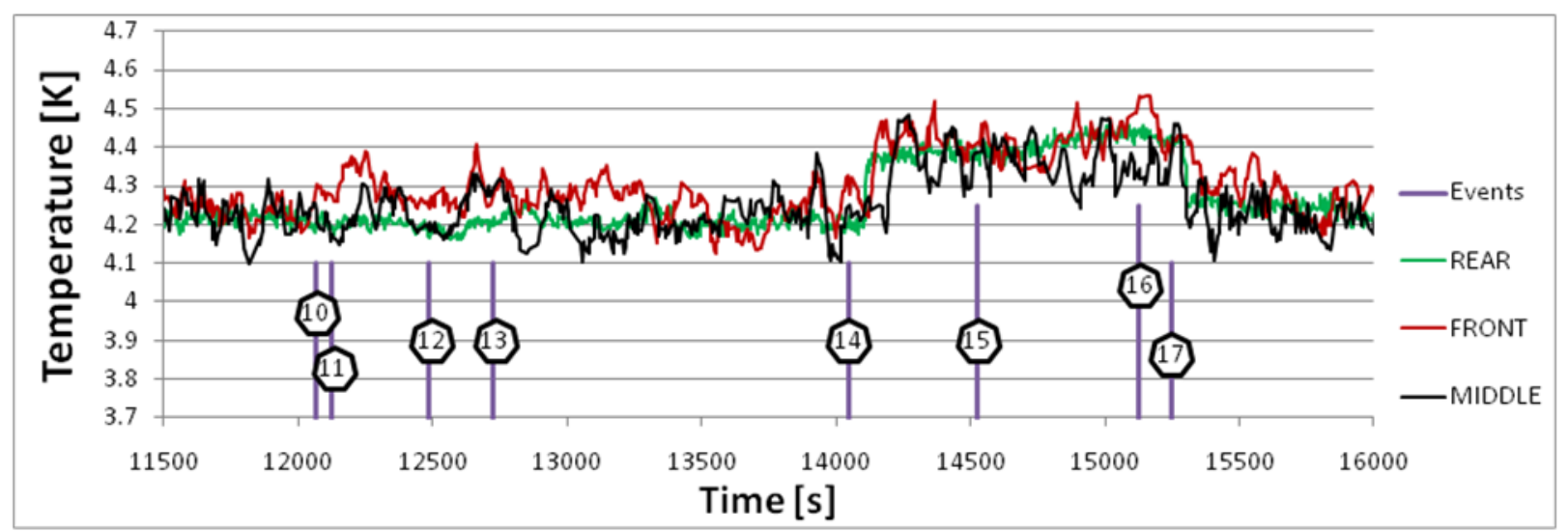

\begin{tabular}{|l|c|c|c|c|c|c|c|c|}
\hline Event Number & 10 & 11 & 12 & 13 & 14 & 15 & 16 & 17 \\
\hline Base heater (\#1) & On & On & & & On & On & On & \\
\hline Bubble heater (\#2) & & On & & & On & On & On & \\
\hline Angle & $-10^{\circ}$ & $-10^{\circ}$ & $-10^{\circ}$ & $10^{\circ}$ & $10^{\circ}$ & $10^{\circ}$ & $10^{\circ}$ & $10^{\circ}$ \\
\hline
\end{tabular}

Figure 6: $2^{\text {nd }}$ series of experiment data. Details are the same as in fig.5

In event \#1, the boat was placed horizontally, and only heater \#1 was turned on. The temperature rose by is about $0.1 \mathrm{~K}$. In the next step, we turned on both heaters; we did not observe any change. We then turned off both heaters and adjusted the boat's angle, tilting the front downwards, to trap more bubbles (event \#4). Heater \#1 was turned on for event \#5; the temperature increased by about $0.15 \mathrm{~K}$. Both heaters were on in event \#6. Similar to event \#2, it was hard to detect any temperature difference due to activating heater \#2. In event \#8, the boat's angle was adjusted to release the trapped bubbles.

Fig. 6 is the continuation of the events depicted in Fig.5. As described, we had adjusted the boat's angle (front up) before event \#10 to release the bubbles. There was an almost negligible rise in temperature increase, probably $0.05 \mathrm{~K}$, even with both heaters working. After event \# 13 , we again changed the angle of the boat to trap bubbles. During event \#14, heater \#1 was on, and the temperature increase was roughly $0.15 \mathrm{~K}$. We adjusted the power from heater $\# 2$ from $20 \mathrm{~W}$ to $2 \mathrm{~W}$ for event $\# 15$, and further changed it gradually from $2 \mathrm{~W}$ to $30 \mathrm{~W}$ in event \#16. Apparently, the bubble from heater \#2 had minimal effect on temperature. After event $\# 17$, we turned off both heaters; the temperature dropped about $0.15 \mathrm{~K}$.

Our video showed that no big bubbles formed at the bubble exit during the test. We saw some, less than $1 \mathrm{~mm}$ in size, at the exit, which heater \#1 had produced. The bubbles generated by heater \#2 were all tiny, micron size like the water droplets in fog.

\section{Discussion:}

Our experiment data is qualitatively consistent with the findings from our model: Thus, with a detrapping angle there are less bubbles, and, therefore, a smaller $\Delta \mathrm{T}$; with a horizontal angle, the bubbles are medium sized, and so there is a medium $\Delta \mathrm{T}$; and, with a trapping angle, more bubbles are 
formed, and then the $\Delta \mathrm{T}$ is higher. However, the temperature change $(\sim 0.15 \mathrm{~K})$ is much smaller than predicted by the model ( $\sim .5 \mathrm{~K})$. As described above, no big bubbles were observed.

The inconsistency was due to the inaccuracy of the original model that assumed the generation of big bubbles. As supported by our videos, no big bubbles were in the trapping region. Several factors are responsible for this. Thus, LH has a very low viscosity, $30 \mu \mathrm{P}$ [1], comparable to that of air ( $19 \mu \mathrm{P})$, and helium gas ( $20 \mu \mathrm{P})$. This low viscosity allows the bubbles to move freely in $\mathrm{LH}$, even those of very small size. As observed in the video, heater \#2 generated fog-like bubbles that flowed quickly in LH. Another critical reason for the formation of small bubbles is the small surface tension of $\mathrm{LH}$; compared to that of water at room temperature, $\approx 70 \times 10^{-3} \mathrm{~N} / \mathrm{m}$, LH has surface tension $\left(\sigma_{\mathrm{LH}}\right)$ of only $0.08 \times 10^{-3} \mathrm{~N} / \mathrm{m}[2]$, a general property of all cryogenic liquids. ) Hence, its contact angle $\theta$ (melting angle) is almost $0^{\circ}$. The bubbles' size, $R_{B}$. on a hot surface facing up is given by [3]

$$
R_{B} \approx f(\theta) \sqrt{\sigma_{L H} / \rho g}
$$

where $f(\theta)$ is function of $\theta, \rho=0.145\left[\mathrm{gram}_{\mathrm{ram}}{ }^{-3}\right]$ is the LH density and g is gravity.

$$
f(\theta) \approx 0.01 \theta \text { when } \theta>>0^{\circ} C[3]
$$

Although equation (5) is not applicable when $\theta$ approaches $0^{\circ}$, it should be a monotonic function of $\theta$. Thus, when the surfaces of Heater \#2 were oriented placed vertically, the radii of the bubbles should not be larger than those evolved when the surfaces face upwards. This is the reason why we saw fog-like tiny bubbles generated by heater \#2 in the video. With the boat's bottom surface facing downward, the surface conditions are quite different from those in equation (4) and heater \#2. This condition helps to build big bubbles and that was why we saw much bigger bubbles at the edge of exit. We It is expectthat big bubbles could be formed especially when the boat's front is pushed down, provided that the $\mathrm{LH}$ is very motionless. However, in our experiments and during the real operation of the $56 \mathrm{MHz}$ cavity. the heat automatically creates $\mathrm{LH}$ flow, viz., another important condition preventing the formation of bubbles .

\section{Conclusions:}

We studied the formation of liquid helium bubbles and their thermal effects on the cavity based on our simplified prototype. Due to the low viscosity of liquid helium and its low surface tension, no big bubbles were observed in the test. The tiny bubbles that were generated by heater \#2 behaved like a light gas travelling in a free space. They quickly escaped from the trapping region. Even those bubbles generated by heater \#1 were not very big ones (although much bigger than the bubbles from heater \#2) due to the liquid flow that was generated by heating. We conclude that the effects of bubbles during the operation of the $56 \mathrm{MHz}$ cavity operation will be negligible.

Acknowledgement:

The authors would like to express their great thanks to Roberto Than, David Pate, and Thomas Tallerico for providing the system design and test support. 
References:

[1] R. Bowers and K. Mendelssohn, "The Viscosity of Liquid Helium between 2 and 5 degrees K", Proc. R. Soc. Lond. A 1950 204, 366-374

[2] R J. Donnelly and CF. Barenghi, "The Observed Properties of Liquid Helium at the Saturated Vapor Pressure", J. Phys. Chem. Ref. Data, Vol. 27, No. 6, 1998, P1245.

[3] Yu. A. Kirichenko, L. A. Slobozhanin and N. S. Shcherbakova, "Detachment Size of Bubbles During Quassistatic Growth on Heater", UDC 532.65:536.423.1 